\title{
Structural features of the aleurone layer of the seed coat associated with imbibition injury in soybean
}

\author{
Kei Sato'), Yutaka Jitsuyama'), Tetsuya Yamada ${ }^{1)}$, Baohui Liu' ${ }^{2,3)}$ and Jun Abe*1) \\ 1) Research Faculty of Agriculture, Hokkaido University, Sapporo, Hokkaido 060-8589, Japan \\ 2) School of Life Sciences, Guangzhou University, Guangzhou 510006, China \\ 3) Key Laboratory of Soybean Molecular Design Breeding, Northeast Institute of Geography and Agroecology, Chinese Academy of \\ Sciences, Harbin 150081, China
}

\begin{abstract}
Soybean (Glycine max) seeds are prone to imbibition injury caused by a rapid uptake of water. Genetic variation in imbibition injury tolerance is well documented, but the underlying mechanisms remain unclear. The aim of this study was to clarify the role of the aleurone layer of seed coat in the tolerance and its structural differences between tolerant and susceptible cultivars. Imbibition injury tolerance was closely related to the water absorption rate of seeds, which was regulated by the aleurone layer of the seed coat. Cryo-scanning electron microscopy analysis revealed that water absorbed in seed coats entered the seed preferentially through the aleurone layer of the top area above the raphe. In susceptible cultivars, the cell walls of the aleurone layer facing the cotyledon in this area were thin and the surface showed shallow depression-like structures, a distinct structure different from those of the tolerant cultivars, which had aleurone cells with thick outer cell walls and smooth and stripe-like deposits. The differences in the structural features of the cell walls and surfaces of aleurone cells in the top area of the seed may be responsible for the difference in the extent of imbibition injury between susceptible and tolerant cultivars.
\end{abstract}

Key Words: Glycine max, soybean, imbibition injury, seed coat, aleurone layer, cryo-SEM, LVP-SEM.

\section{Introduction}

Regulation of the moisture content of harvested seeds is critical for the retention of viability during storage and germinability in the field. Soybean (Glycine max) seeds are vulnerable to structural and functional injury caused by rapid imbibition. Imbibition injury results in poor and unstable seedling emergence, particularly in fields with excess moisture caused by heavy or continuous rainfall and in those converted from paddy fields with poor drainage (Kokubun 2013). Imbibition injury also negatively affects food processing (Koizumi et al. 2008, Saio 1976). It occurs only in seeds with low moisture contents (Hobbs and Obendorf 1972, Jitsuyama et al. 2014, Toledo et al. 2010, Vertucci and Leopold 1984). An osmopriming treatment of seeds before seeding is thus proposed as an effective procedure to alleviate imbibition injury (Kokubun 2013).

Genetic variation in the degree of imbibition injury is

Communicated by Toyoaki Anai

Received November 13, 2018. Accepted March 26, 2019.

First Published Online in J-STAGE on May 18, 2019.

*Corresponding author (e-mail: jabe@res.agr.hokudai.ac.jp) well documented (Chachalis and Smith 2000, Hou and Thseng 1991, Jitsuyama et al. 2014, Muramatsu et al. 2008, Nakajima et al. 2015, Raymond et al. 1981, Shao et al. 2007, Tian et al. 2005). Seeds with slower absorption show higher tolerance to injury than seeds with faster absorption (Chachalis and Smith 2000, Muramatsu et al. 2008, Ragus 1987). Seed-coat permeability to water, which is controlled by the outmost layer of the seed coat (cuticle), has been investigated as a regulator for water entry into embryos (reviewed by Qutob et al. 2008). In permeable seeds, the cuticle has multiple small cracks, some of which reach the palisade layer to form an unobstructed route of water entry into the seed coat, whereas no such cracks are present in impermeable seeds (Jang et al. 2015, Ma et al. 2004). The cuticle of impermeable seeds is rich in hemi-cellulose (Mullin and Xu 2001), hydroxylated fatty acids (Shao et al. 2007), $\beta$-1,4-glucan derivatives (Jang et al. 2015), and calcium (Saio 1976, Sun et al. 2015), all these properties contribute to the structural rigidity needed to inhibit water entry into the seed coat. The molecular basis of the quantitative trait loci for impermeability has been identified (Jang et al. 2015, Sun et al. 2015).

The tolerance to imbibition injury varies even among 
cultivars with permeable seed coats, and is associated with the variation in water absorption rate (Chachalis and Smith 2000, Hou and Thseng 1991, Jitsuyama et al. 2014, Ragus 1987, Raymond et al. 1981, Tian et al. 2005). Because soybean seeds have no specific site for water entry, water enters seed coats through various areas, such as the hilum, raphe, microphyle, and minute cracks on the dorsal side of seeds, although the first site of water entry varies with cultivars (Koizumi and Kano 2014, Ma et al. 2004, Meyer et al. 2007, Muramatsu et al. 2008, Shao et al. 2007, Tian et al. 2005). Water absorption rate is also controlled by the osmolarity of embryos (Jitsuyama et al. 2014) and by the aleurone layer, the innermost layer of the seed coat (Matsui et al. 1996); embryos with the aleurone layer removed rapidly collapsed or lost germinability by a rapid imbibition of water. Tian et al. (2005) reported that the aleurone layer regulates water entry into embryos regardless of the degree of cultivar tolerance to imbibition injury. The aleurone layer may therefore be another regulator for water entry to confer tolerance to injury that is caused by rapid imbibition. Matsui et al. $(1995,1996)$ found that the cell walls of soybean aleurone cells contain a specific glycine-rich protein and suggested its role in the regulation of water entry into the embryo. However, it remains unknown what structures and functions of aleurone cells are responsible for the differences in imbibition injury tolerances among cultivars.

In this study, we investigated the structure of the cell walls of the aleurone layer facing the cotyledons in soybean cultivars tolerant and susceptible to imbibition injury. Our results suggest the structural features that underlie the differences in injury tolerance.

\section{Materials and Methods}

\section{Plant materials}

We used two Glycine max cultivars tolerant to imbibition injury, the Japanese cultivar Hayahikari and the Korean accession NK07, and two susceptible Japanese cultivars, Toyomusume and Tachinagaha. The seed coat was yellow in Hayahikari, Toyomusume, and Tachinagaha and pale-green in NK07. The 100-seed weight was ca. $20 \mathrm{~g}$ in Hayahikari, $35 \mathrm{~g}$ in Toyomusume and Tachinagaha, and $10 \mathrm{~g}$ in NK07. Toyomusume, Hayahikari, and NK07 matured early and were cultivated in an experimental field of Hokkaido University, Sapporo, Japan (lat. $43^{\circ} 08^{\prime} \mathrm{N}$, long. $141^{\circ} 34^{\prime} \mathrm{E}$ ). Tachinagaha, a cultivar from the central Honshu region of Japan, was grown in a greenhouse. Seeds harvested in 2012 to 2014 were used in this study. All seeds were dried in a desiccator for 3 weeks to lower the moisture content.

\section{Water absorption rate}

Water absorption rate of seeds incubated under water or polyethylene-glycol (PEG) 1000 (Wako, Tokyo, Japan) solutions $(10 \%, 20 \%$, and $40 \%)$ was examined for Toyomusume and Hayahikari. Ten seeds were first weighed on an electronic balance (sensitivity, $0.01 \mathrm{mg}$; R180D, Sartorius AG,
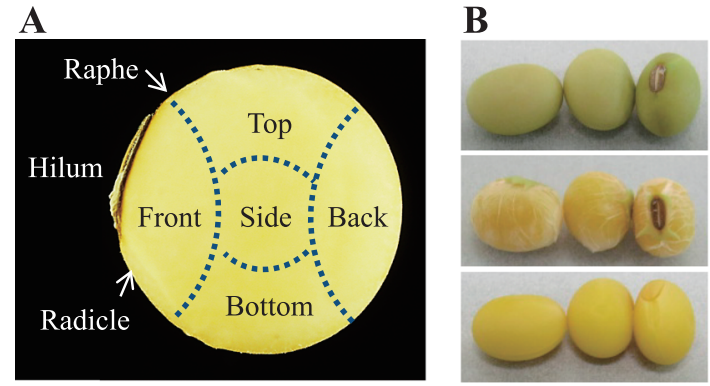

C

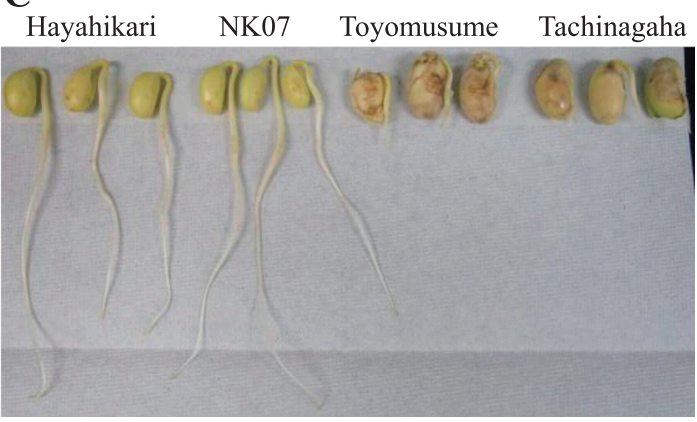

Fig. 1. Five areas of soybean seed compared for seed coat characteristics, cotyledon with or without aleurone layer, and imbibition injury among cultivars. (A) Five areas of soybean seed coat compared in terms of water absorption rate and structural features of the aleurone layer. (B) Intact seeds of NK07 with pale green seed coats (top panel), cotyledons covered by aleurone layer (middle panel), and naked cotyledons (bottom panel). (C) Imbibition injury of sensitive and tolerant cultivars. Photo was taken three days after the 24-h immersion in distilled water.

Gottingen, Germany). The seeds were immersed in distilled water or PEG solution and were weighed at 10, 30, and $60 \mathrm{~min}$, and then every hour until 6 or $9 \mathrm{~h}$; before weighing, water on the seed coat was carefully wiped off with a paper towel. After treatments, the seeds were dried in an electronic oven $\left(80^{\circ} \mathrm{C}\right)$ for 2 days and weighed. The amount of water absorbed by each time point was calculated per seed dry weight. Water absorption rates per dry weight $(\mathrm{mg} / \mathrm{g} / \mathrm{min})$ were expressed with the linear regression coefficients of the amount of water absorbed as a function of time after immersion (min). The experiments were performed in triplicate (10 seeds per replicate).

To determine whether the rate differed among different areas of the seed coat, the amount of water absorbed in 10 min after immersion was determined separately for each of the five areas: front (including the area flanking the hilum), either of two side areas (abaxial), back (dorsal), bottom, and top (Fig. 1A). Dried seeds, except the targeted area, were pre-coated with water-repellent resin (silicone lacquer spray, Kanpe-hapio Co., Ltd., Osaka, Japan). The experiments were repeated four times (10 seeds per replicate).

\section{Imbibition injury}

Imbibition injury was evaluated as follows. Ten seeds 
were packed in a nylon mesh bag $(95 \times 70 \mathrm{~mm})$ and immersed in distilled water at room temperature for $24 \mathrm{~h}$. Water on the seed coat was wiped off with a paper towel; the seeds were arranged along a straight line at about fourfifths from the bottom on one side of two-folded germination paper $(220 \times 230$ mm; Cherry, Sinbashi Seishi, Fuji, Japan) and attached with sticky tape. The folded paper was rolled, fastened loosely with a wire, and placed upright in a box containing the minimum amount of water sufficient to wet the paper. The box was covered with aluminium foil to avoid desiccation. Three days later, the length of the radicle was measured for each seed, and percentage of normally germinated seeds (those with a radicle of more than $3 \mathrm{~cm}$ ) was used as an index of imbibition injury. Imbibition injury was also examined in seeds with a moisture content of $\geq 10 \%$ and seeds whose coats were stripped wholly or partly. The moisture content of seeds was elevated by keeping them in a high-humidity box at room temperature for one week. The seed coat was removed either entirely (with the aleurone layer) or only the outer layer (palisade to parenchyma cells) was removed and the aleurone layer was left intact (Fig. 1B). Stripping was performed over the entire seed surface or only in a circular area of ca. 3-mm diameter on a side area of the seed. In the former case, seeds were first immersed in distilled water for a short time until wrinkles were formed in the surface, and then the outer layer or entire seed coat was removed with sharp anatomical forceps carefully. The stripped seeds were dried in a desiccator for at least one week before the germination test. The outer layer or entire seed coat was exercised in a circular with a razor from dried seeds. All experiments were performed in triplicate (10 seeds per replicate).

\section{Statistical procedure}

Treatment/cultivar means were statistically tested by F-test or Tukey-Kramer multiple comparison procedure.

Low vacuum pressure scanning electron microscopy (LVP-SEM) analysis

Structures of the surface of the aleurone layer facing the cotyledons in the four cultivars were observed by LVPSEM. Five dried seeds per cultivar were dehydrated in an ethanol series (50-100\%) and 97\% isoamyl acetate solutions, and were completely dried with a critical-point dryer (HCP-2, Hitachi Ltd., Tokyo, Japan). The seeds were divided into five areas (Fig. 1A); the internal surface (ca. $25 \mathrm{~mm}^{2}$ ) of the aleurone layer from each area was mounted upward on the metal stage of a SEM (JSM-5310LV, JEOL Ltd., Tokyo, Japan) and coated with platinum particles by using an ion sputter (E101, Hitachi Ltd.). All samples were observed with the SEM at $15 \mathrm{kV}$.

\section{Cryo-scanning electron microscopy (cryo-SEM) analysis}

Dried seeds of Toyomusume and Hayahikari were immersed in water for $3 \mathrm{~h}$ and excess water on the surface was removed. The seeds were attached to sample stages by an adhesive agent with the side area of the seed facing upward and then rapidly frozen in liquid nitrogen. The frozen sample was set on a cold stage of cryo-SEM (JSM-6701F, JEOL Ltd.), which had been pre-cooled to $-130^{\circ} \mathrm{C}$, and then the temperature was increased to $-110^{\circ} \mathrm{C}$ and maintained for $5 \mathrm{~min}$. The seed was cut horizontally with a razor to separate the cotyledons from each other, and the upper cotyledon was removed. The temperature of the seed was increased to $-95^{\circ} \mathrm{C}$, and the surface of the specimen was coated with gold/palladium for $3 \mathrm{~min}$. The presence of ice crystals (signs of imbibed water) on the surface of the aleurone layer was examined in the top and back areas of the seeds. Five seeds per cultivar were used. Six to ten SEM images per seed were taken for each side.

Calcofluor white staining of the inner and outer cell walls of aleurone cells

Seed coat samples excised from the top and back areas of Toyomusume and Hayahikari seeds were embedded into 5\% agar and sliced into sections $(30 \mu \mathrm{m})$ with a micro-slicer (VT-1200 S, Leica Biosystems, Nussloch, Germany). The sections were stained with $1 \mathrm{mg} / \mathrm{L}$ calcofluor white M2R solution (Polysciences, Inc., Warrington, PA, USA) and examined under UV illumination on a confocal microscope (Leica TCS-SP5, Leica Microsystems, Tokyo, Japan). Five seeds per cultivar were used.

\section{Results}

Regulation of water entry into the embryo by the aleurone layer

After 24-h imbibition treatment of dried seeds, in most of the seeds of susceptible cultivars (Toyomusume and Tachinagaha) cotyledons partly decayed or the growth of radicles ceased, whereas seeds of imbibition injury-tolerant cultivars (Hayahikari and NK07) developed normal radicles (Fig. 1C). When the moisture content was elevated to $\geq 10 \%$, the percentages of normally germinated seeds in the susceptible cultivars were increased to levels similar to those of the tolerant ones (Fig. 2A), suggesting that imbibition injury of susceptible cultivars was caused by the lower moisture content in seeds. Furthermore, in seeds with high moisture content, the completely exposed embryos without seed coats germinated normally (Fig. 2A). These results were consistent with those in previous reports (Jitsuyama et al. 2014, Toledo et al. 2010), suggesting that imbibition injury observed in susceptible cultivars was caused by rapid water absorption by seeds with low moisture contents.

We compared the water absorption rate in different areas of seeds between Toyomusume and Hayahikari. In Toyomusume, water easily entered the seed coat through all areas except for the front area, in contrast to Hayahikari, in which water entered the seed coat mainly through the back area (Fig. 2B). Consequently, water imbibition in whole seeds was faster in Toyomusume than in Hayahikari (Fig. 2C). The water absorption rate was $2.9 \mathrm{mg} / \mathrm{g} / \mathrm{min}$ in 

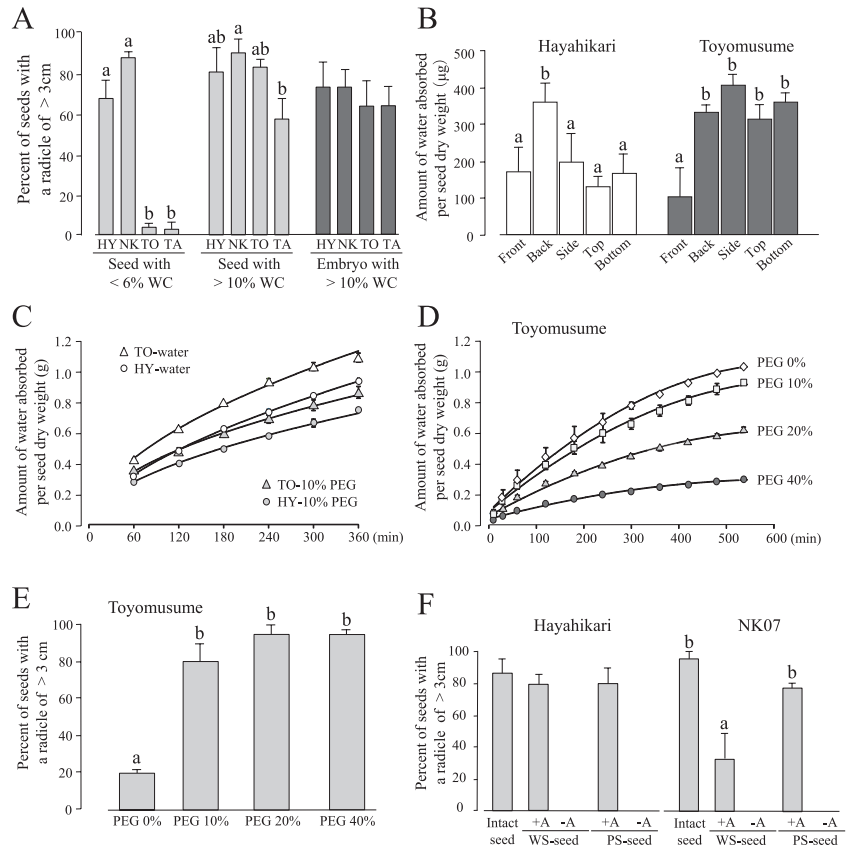

Fig. 2. Imbibition injury, water absorption, and role of aleurone layer in imbibition-injury sensitive and tolerant cultivars. (A) Percent of seeds with a radicle of $>3 \mathrm{~cm}$ in soybean seeds and cotyledons with different moisture contents after 24-h imbibition under submergence. (B) Water absorption in the five areas of seed coats in Hayahikari (open bars) and Toyomusume (closed bars). (C) Time course of changes in amount of water absorbed per seed dry weight for Hayahikari and Toyomusume. Water absorption rates were 2.9 and $2.4 \mathrm{mg} / \mathrm{g} / \mathrm{min}$ in water and 2.1 and $1.8 \mathrm{mg} / \mathrm{g} / \mathrm{min}$ in the $10 \%$-PEG solution in Toyomusume and Hayahikari, respectively. (D) Time course of changes in the amount of water absorbed per seed dry weight in PEG solutions of different concentrations in Toyomusume. (E) Imbibition injury of Toyomusume seeds after 24-h immersion in water and PEG solutions. (F) Percent of seeds with a radicle of $>3 \mathrm{~cm}$ in seeds with or without aleurone layer $(+\mathrm{A} /-\mathrm{A})$. Seed coats were wholly (WS) or partly (PS) stripped from embryos. The Tukey-Kramer test was carried out (A) among cultivars in each of different water contents, (B) among five areas in each cultivar, (E) among different PEG concentrations, and (F) among different seed coat treatments in each cultivar. Different alphabets indicate that mean values were significantly different at $5 \%$ level (A, B and F) and 1\% level (E). HY, Hayahikari; NK, NK07; TO, Toyomusume; TA, Tachinagaha; WC, water content.

Toyomusume and $2.4 \mathrm{mg} / \mathrm{g} / \mathrm{min}$ in Hayahikari. To determine the effects of water absorption speeds on the injury, PEG was used to generate different water availability in imbibition. Water absorption in Toyomusume seeds was suppressed in a PEG concentration-dependent manner (Fig. 2D). Water absorption rates were $2.6,2.0,1.4$ and $0.6 \mathrm{mg} / \mathrm{g} / \mathrm{min}$ in $0 \%$, $10 \%, 20 \%$ and $40 \%$-PEG solutions, respectively. The degree of imbibition injury was significantly (at $1 \%$ level) lower in seeds immersed in PEG solutions than those in water (Fig. 2E); even 10\% PEG increased the percentage of normally germinated seeds almost to the level of tolerant cultivars (Fig. 2A). The water absorption rate of Toyomusume seeds in $10 \%$ PEG solution $(2.1 \mathrm{mg} / \mathrm{g} / \mathrm{min})$ was slightly lower than that in Hayahikari seeds in water (Fig. 2C), suggesting that a gradual uptake of water by cotyledons relieved the functional disruption of dried cotyledons due to rapid imbibition.

To determine the role of the aleurone layer in the control of water entry into embryos, we examined germination after 24-h imbibition in seeds of tolerant cultivars whose seed coats were stripped wholly or partly with the aleurone layer removed or intact. Regardless of the stripped area, all seeds without the aleurone layer collapsed rapidly during imbibition or ceased radicle growths in both cultivars (Fig. 2F). In contrast, embryos of Hayahikari with the intact aleurone layer retained normal radicle growth, which was similar to that of intact seeds; the percentage of normally germinated seeds were almost the same between the intact seeds and wholly- or partly-stripped seeds. The tolerance to imbibition injury was also observed in the partly-stripped seeds in NK07, although the percentage of normally germinated seeds was significantly lowered in the wholly-stripped seeds (Fig. 2F).

\section{Cryo-SEM analyses of the dynamics of water imbibed by the seed coat}

To determine how water absorbed by the seed coat entered the seeds, we used cryo-SEM to examine the distribution of ice crystals (signs of absorbed water) on the surface of the aleurone layer facing the cotyledons in seeds immersed in water for $3 \mathrm{~h}$. In the back area of Toyomusume seeds, ice crystals were not frequently observed in spaces between the wrinkled seed coat and cotyledons (Fig. 3A), although they were occasionally formed on the surface of the aleurone layer (Fig. 3B). Ice crystals were also rarely observed in the back area of Hayahikari seeds (Fig. 3C). In contrast, large masses of ice crystals were frequently formed in the top areas of Toyomusume seeds (Fig. 3D). Such masses of ice crystals, however, were rarely formed in the top area of Hayahikari seeds; small papillary ice crystals were occasionally formed in a cluster (Fig. 3E, 3F). These findings suggest that water absorbed by seed coats did not enter the seeds evenly through all areas, but rather preferentially from the top of the seeds in the susceptible cultivar Toyomusume.

\section{Characteristics of the surface structure of the aleurone layer}

We used LVP-SEM to compare the structure of the aleurone layer surface facing the cotyledons between susceptible and tolerant cultivars. Two types of surface structures were common in all areas, except the seed top in the susceptible cultivars: one composed of aleurone cells with clearly discernible cell walls (Asterisks, Fig. 4) and the other one covered by smooth and stripe-like deposits (Fig. 4C, 4D). Small pits between aleurone cells, particularly in the side areas (Arrows, Fig. 4A), were observed in all the tolerant and susceptible cultivars tested; therefore, these pits did not contribute to the difference in imbibition injury among the 

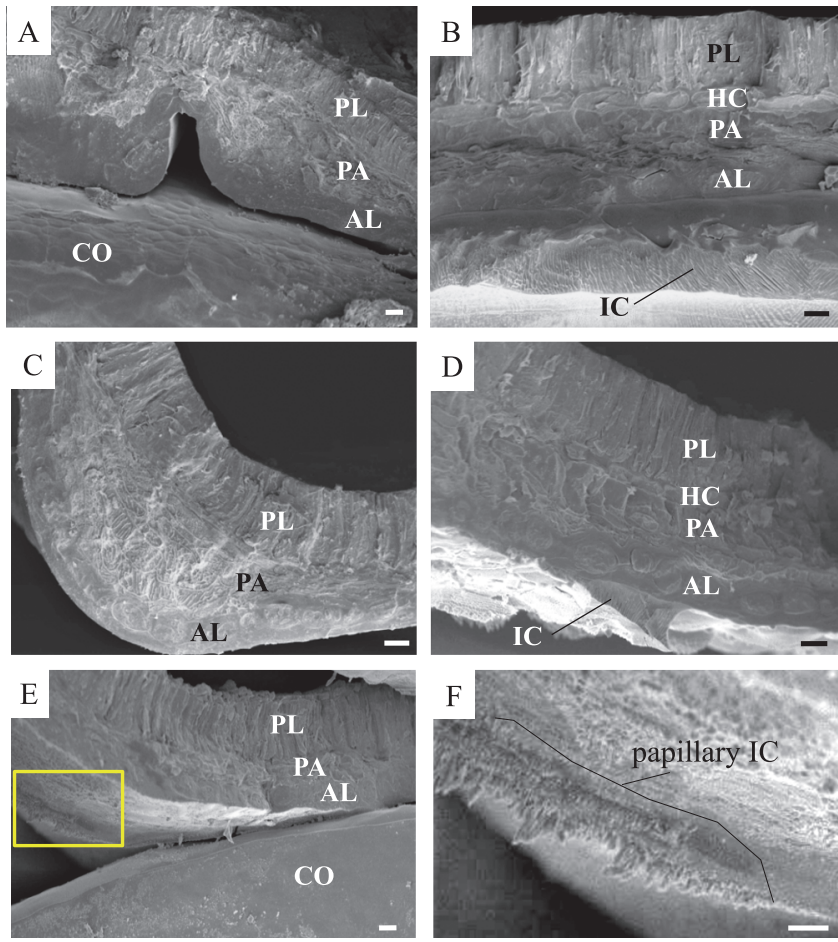

Fig. 3. Ice crystals formed on aleurone layer surfaces facing the cotyledons on the back (dorsal) and top areas of soybean seeds. Seeds imbibed for $3 \mathrm{~h}$ were rapidly frozen in liquid nitrogen. The distribution of ice crystals was observed by cryo-SEM. (A) and (B) Back areas of Toyomusume. (C) Back area of Hayahikari. (D) Top area of Toyomusume. (E) Top area of Hayahikari. (F) A magnified view of the area outlined in yellow in (E). PL, palisade layer; PA, parenchyma cells; $\mathrm{HC}$, hourglass cells; AL, aleurone layer; $\mathrm{CO}$, cotyledon; IC, ice crystals. Bars, $10 \mu \mathrm{m}$.

cultivars. In the top areas, surface structure of the aleurone layer was clearly different between Hayahikari and Toyomusume. The aleurone layer of Hayahikari was covered with smooth and stripe-like deposits (Fig. 4D), as observed in the other areas, whereas that of the Toyomusume was composed of shallow depression-like aleurone cells of different shapes (Fig. 4E). The surface of the aleurone layer in the top areas of seeds was not flat, but rather wavy in both tolerant and susceptible cultivars (Arrowheads, Fig. 4D, 4E), unlike the surfaces in the other parts (Fig. 4A, 4B). The similar characteristics in the structure were also observed in the imbibition-injury sensitive Tachinagaha and tolerant NK07. In the top areas of the seeds, Tachinagaha exhibited shallow depression-like aleurone cells of different shapes, whereas in NK07 the aleurone layer was covered with smooth and stripe-like deposits (Fig. 5).

\section{Calcofluor white staining of the inner and outer cell walls of aleurone cells}

Calcofluor-white binds to $\beta$-1,4-glucan to generate fluorescence. Jang et al. (2015) found that $\beta$-1,4-glucan accumulates in the outer and inner layers of aleurone cells in soybean seeds. We used calcofluor white staining to exam-
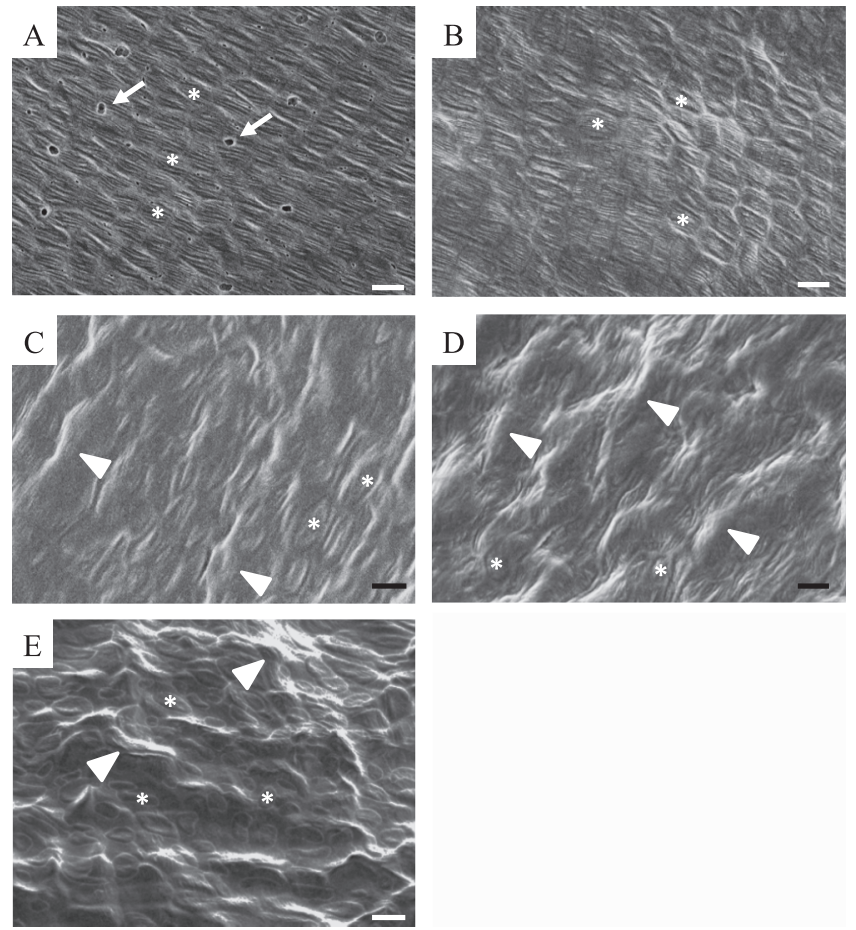

Fig. 4. Structure of the aleurone layer surface facing the cotyledons in various areas of soybean seeds. (A) and (B) Side and bottom areas of Toyomusume, (C) Front area of Hayahikari, (D) and (E) Top areas of (D) Hayahikari and (E) Toyomusume. Asterisk, shallow depressionlike aleurone cell; Arrow head, wavy structure; Arrow, pit. Bars, $10 \mu \mathrm{m}$.

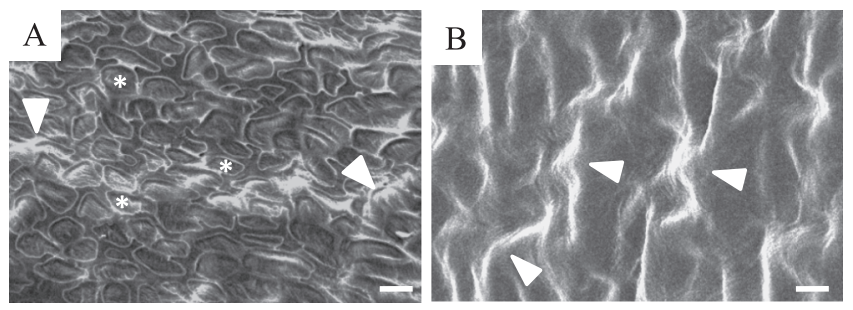

Fig. 5. Structure of the aleurone layer surface facing the cotyledons in various areas of soybean seeds. (A) Tachinagaha, (B) NK07. Asterisk, shallow depression-like aleurone cell; Arrow head, wavy structure; Arrow, pit. Bars, $10 \mu \mathrm{m}$.

ine the accumulation of $\beta$-1,4-glucan in cell walls of the aleurone layer of Toyomusume and Hayahikari. Fig. 6 shows transmission images for a cross-section of seed coat merged with the blue-fluorescent images after calcofluorwhite staining. Fluorescence was detected in palisade cells, hourglass cells, and the inner and outer layers of aleurone cells (Fig. 6A, 6B), in agreement with Jang et al. (2015). There was no difference in fluorescence intensity at any positions between Toyomusume and Hayahikari. However, the cell walls of the outer layer facing the cotyledons were thicker in Hayahikari than Toyomusume (Fig. 6A, 6B, 6D). The aleurone layer was thicker in the top of seeds than in the back in both cultivars, although the difference was significant at $5 \%$ only in Hayahikari by Tukey-Kramer test (Fig. 6C). Cell wall thickness in the outer layer was significantly 
A Hayahikari
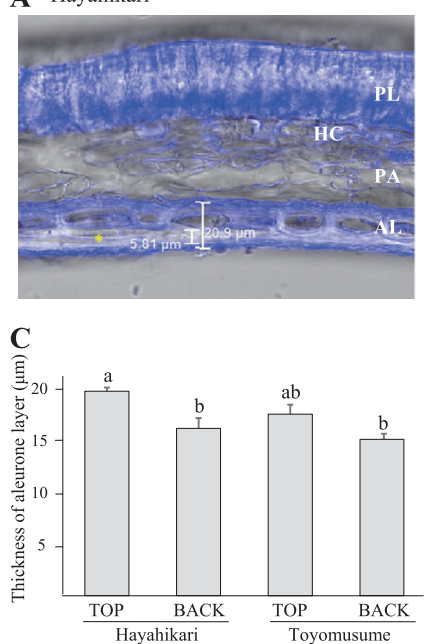

B Toyomusume
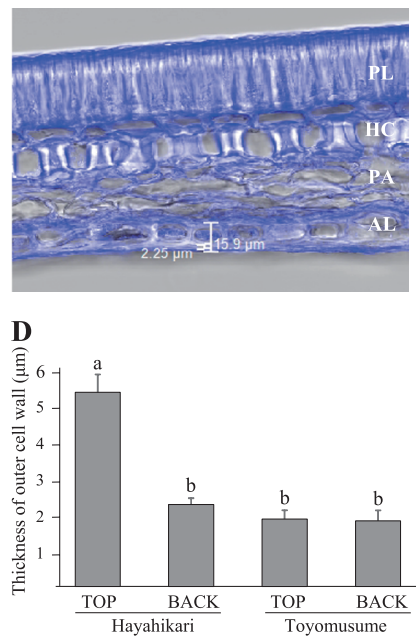

Fig. 6. Thickness of the aleurone layer and cell walls in the aleurone layer in the top and back areas of soybean seeds. (A) and (B) Crosssections of the seed coat in the top area in (A) Hayahikari and (B) Toyomusume. (C) Thickness of the aleurone layer. (D) Thickness of the outer cell wall in the aleurone layer. Asterisk, thickened structure in the outer cell wall. Different alphabets indicate significant difference at $5 \%$ between mean values by Tukey-Kramer test. PL, palisade layer; PA, parenchyma cells; HC, hourglass cells; AL, aleurone layer

greater in Hayahikari than Toyomusume at the top of seeds, but was almost the same in both cultivars at the back (Fig. 6D).

\section{Discussion}

This study demonstrated that imbibition injury in soybean seeds is caused by a rapid uptake of water by dried seeds and that the aleurone layer plays a key role in tolerance, in line with previous studies (Matsui et al. 1996, Tian et al. 2005). In all areas of the seed surface except the front area, the seed coat of Toyomusume absorbed water at high rates; in contrast, in Hayahikari water entered mainly through the back area (Fig. 2B). The difference between the two cultivars in the water absorption rate at the early stage of imbibition (Fig. 2C) may therefore reflect the difference in the water absorption patterns of seed coats. Easy water absorption by seed coats in Toyomusume might be ascribed to the presence of minute cracks in the cuticle, similar to those reported in the back area of seeds in Tachinagaha (Jang et al. 2015). Such cracks might be distributed over the whole seed coat in Toyomusume, although we did not examine the structure of the cuticle surface. The susceptibility of Toyomusume to injury was alleviated to a level similar to that in Hayahikari by suppressing water absorption by PEG solutions (Fig. 2C, 2D). Reinforcement of the cuticle structure to reduce permeability may therefore be a means to improve the tolerance of Toyomusume.

Although Hayahikari had a slower water uptake than Toyomusume, its tolerance may be ascribed not to the grad- ual entry of water into seed coats, but rather to the control of water entry by the aleurone layer, because the tolerance was retained only if the aleurone layer remained overlaid on the embryo, even when the outer layer of the seed coat (from the cuticle to parenchyma cells) was removed (Fig. 2F). The role of the aleurone layer in the tolerance of soybean seeds to imbibition injury was previously demonstrated by Matsui et al. (1996) and Tian et al. (2005). In this study, we demonstrate that differences in the structure and/or function of the cuticle and aleurone cells may be involved in the differences in the degree of imbibition injury among cultivars.

Using micro-magnetic resonance analysis of a blackseeded soybean cultivar, Koizumi et al. (2008) found that water enters the seed coat through the raphe, rapidly spreads to the opposite side of the hilum, and then diffuses all over the seed coat. Water is temporarily stored in the seed coat and eventually enters the inner part of the seed (Koizumi et al. 2008, Koizumi and Kano 2014). Cryo-SEM analysis was used in this study to visualize the dynamics of water absorbed in seeds immersed in water for $3 \mathrm{~h}$. We selected the back area of seeds in which water entered the seed coat easily in both Toyomusume and Hayahikari (Fig. 2B) and the top area in which the surface structure of aleurone layer facing the cotyledon was distinctly different between the two cultivars (Fig. 4) as representative areas for the analysis. In Toyomusume, large masses of ice crystals frequently formed on the surface of aleurone layer facing the cotyledon in the top area of seeds, although they were occasionally observed in the back area. In contrast, only small papillary crystals were found in Hayahikari; ice crystals were rarely observed in the back area. The ice crystals observed were thus not any experimental artifacts produced during processing of samples expected to be randomly generated irrespective of areas and cultivars. The results obtained in Cryo-SEM analysis suggest that, in both Toyomusume and Hayahikari, the water absorbed by the seed coat entered the seed preferentially from the top area compared with the back of seeds. Different patterns of ice crystal formation were thus consistent with different rates of water absorption between the two cultivars (Fig. 2C).

We found a distinctive difference between tolerant and susceptible cultivars in the structure of the surface of the aleurone layer facing the cotyledons, particularly in the top area of seeds. In the tolerant cultivars, Hayahikari and NK07, aleurone cells had clearly discernible cell walls or were overlaid by smooth and stripe-like deposits; similar structures were observed in all seed areas (Figs. 4, 5). In the top areas of the seeds of susceptible cultivars, shallow depression-like aleurone cells were observed, but no such structures were observed in the tolerant cultivars or on the surfaces of other seed parts in the susceptible cultivars. The surfaces of the aleurone layer in the top area were wavy in all four cultivars (Figs. 4, 5). The surface structures of the aleurone cells in the top area may be affected by cotyledon expansion until physiological maturity and then reduction because of dehydration in the late stage of maturation. In the 
top area, the cell walls of the aleurone layer facing the cotyledons were significantly thicker in Hayahikari than Toyomusume, whereas there was no difference in the back area (Fig. 5). Thick outer cell walls of aleurone cells in the top area may play a critical role in protection from injury caused by rapid water imbibition by dried seeds. Further study is needed to determine whether the cell wall thickening observed in Hayahikari is a common mechanism among imbibition injury-tolerant cultivars.

In summary, our findings suggest that the differences in the structural features of cell walls of aleurone cells facing the cotyledons in the top area of seeds is a key factor responsible for different responses of susceptible and tolerant cultivars to imbibition. In susceptible cultivars, water may enter the seed easily and rapidly through unobstructed routes in the cuticle and dysfunctional or physically defective aleurone cells, resulting in embryo deterioration. Our findings may be helpful for further studies on genetic and molecular mechanisms involved in the structure and function of the aleurone layer in imbibition-injury-tolerant cultivars. Such studies would be needed to improve the tolerance of soybean cultivars to imbibition injury.

\section{Acknowledgments}

We thank T. Itoh and M. Yasui for technical assistance in SEM and confocal microscopy. This work was supported partly by a grant from the Ministry of Agriculture, Forestry and Fisheries, Japan (Genomics-based Technology for Agricultural Improvement, SFC-1004) to J. Abe, and by Japan Society for the Promotion of Science KAKENHI (grant 26450015) to Y. Jitsuyama.

\section{Literature Cited}

Chachalis, D. and M.L. Smith (2000) Imbibition behaviour of soybean (Glycine $\max (\mathrm{L}$.) Merrill) accessions with different testa characteristics. Seed Science and Technology 28: 321-331.

Hobbs, P.R. and R.L. Obendorf (1972) Interaction of initial seed moisture and imbibitional temperature on germination and productivity of soybean. Crop Sci. 12: 664-667.

Hou, F.F. and F.S. Thseng (1991) Studies on the flooding tolerance of soybean seed—varietal differences. Euphytica 57: 169-173.

Jang, S.J., M. Sato, K. Sato, Y. Jitsuyama, K. Fujino, H.Mori, R. Takahashi, E.R. Benitez, B.Liu, T. Yamada et al. (2015) A singlenucleotide polymorphism in an endo-1,4- $\beta$-glucanase gene controls seed coat permeability in soybean. PLoS ONE 10: e0128527.

Jitsuyama, Y., Y. Hagihara and Y. Konno (2014) Two imbibition properties independently influence the cultivar-specific flooding tolerance of dried soybean seeds. Seed Sci. Res. 24: 37-48.

Koizumi, M., K. Kikuchi, S. Isobe, N. Ishida, S.Naito and H.Kano
(2008) Role of seed coat in imbibing soybean seeds observed by micro-magnetic resonance imaging. Ann. Bot. 102: 343-352.

Koizumi, M. and H. Kano (2014) Water entry in dry soybeans at imbibition observed by dedicated micro-magnetic resonance imaging. American Journal of Biology and Life Sciences 2: 6-11.

Kokubun, M. (2013) Genetic and cultural improvement of soybean for waterlogged conditions in Asia. Field Crops Res. 152: 3-7.

Ma,F.S., E. Cholewa, T.Mohamed, C.A.Peterson and M.Gijzen (2004) Cracks in the palisade cuticle of soybean seed coats correlate with their permeability to water. Ann. Bot. 94: 213-228.

Matsui, M., I. Toyosawa and M.Fukuda (1995) Purification and characterization of a glycine-rich protein from the aleurone layer of soybean seeds. Biosci. Biotech. Biochem. 59: 2231-2234.

Matsui, M., T. Uenaka, I. Toyosawa and M. Fukuda (1996) Role of soybean aleurone layer in water uptake in seeds. Nippon Nogeikagaku Kaishi 70: 663-669.

Meyer, C.J., E. Steudle and C.A.Peterson (2007) Patterns and kinetics of water uptake by soybean seeds. J. Exp. Bot. 58: 717-732.

Mullin, W.J. and W.Xu (2001) Study of soybean seed coat components and their relationship to water absorption. J. Agric. Food Chem. 49: 5331-5335.

Muramatsu, N., M. Kokubun and A. Horigane (2008) Relation of seed structures to soybean cultivar difference in pre-germination flooding tolerance. Plant Prod. Sci. 11: 434-439.

Nakajima, T., A. Seino, T. Nakamura, Y. Goto and M. Kokubun (2015) Does pre-germination flooding-tolerant soybean cultivar germinate better under hypoxia conditions? Plant Prod. Sci. 18: 146-153.

Qutob, D., F. Ma, C.A.Peterson, M.A. Bernards and M. Gijzen (2008) Structural and permeability properties of the soybean seed coat. Botany 86: 219-227.

Ragus, L. (1987) Role of water absorbing capacity in soybean germination and seedling vigour. Seed Science and Technology 15: 285296.

Raymond,E., M.E. Musgrave and A.C.Leopold (1981) The seed coat as a control of imbibitional chilling injury. Crop Sci. 21: 312-317.

Saio, K. (1976) Soybeans resistant to water absorption. Cereal Foods World 21: 168-173.

Shao, S., C.J.Meyer, F.Ma, C.A.Peterson and M.A.Bernards (2007) The outermost cuticle of soybean seeds: chemical composition and function during imbibition. J. Exp. Bot. 58: 1071-1082.

Sun, L., Z.Miao, C.Cai, D.Zhang, M.Zhao, Y.Wu, X.Zhang, S.A. Swarm, L.Zhou, Z.J.Zhang et al. (2015) GmHs1-1, encoding a calcineurin-like protein, controls hard-seededness in soybean. Nat. Genet. 47: 939-943.

Tian, X.H., T. Nakamura and M.Kokubun (2005) The role of seed structure and oxygen responsiveness in pre-germination flooding tolerance of soybean cultivars. Plant Prod. Sci. 8: 157-165.

Toledo,M.Z., C.Cavariani, J.deB. França-Neto and J.Nakagawa (2010) Imbibition damage in soybean seeds as affected by initial moisture content, cultivar and production location. Seed Science and Technology 38: 399-708.

Vertucci, C.W. and A.C.Leopold (1984) Bound water in soybean seed and its relation to respiration and imbibitional damage. Plant Physiol. 75: 114-117. 